\title{
EVERY FINITE LATTICE IS A FACE LATTICE
}

\author{
BY MARY KATHERINE BENNETT
}

Communicated by Paul R. Halmos, February 9, 1977

ABSTRACT. Given a finite lattice $L$, there is a finite-dimensional convex set $C$ such that $L$ is the lattice of faces of $C$.

If $C$ is a convex set, we denote by $L(C)$ the lattice of convex subsets of $C$. The lattice of (algebraic) faces of $C$ is available from $L(C)$ as $\{x \in L(C):(a \vee b)$ $\wedge x=(a \wedge x) \vee(b \wedge x)$ for all $a, b$ in $L(C)\}$. We shall call this face lattice $D L(C)$. It is a complete meet-sublattice of $L(C)$ and as such induces a closure operator $D$ on $L(C)[1]$.

LEMMA 1. Let $D L(C)$ be finite. If $M$ is a join-sublattice of $D L(C)$ such that $O_{D L(C)}$ is in $M$, then $M$ is isomorphic to $D L\left(C^{\prime}\right)$ for some subset $C^{\prime}$ of $C$.

Here $C^{\prime}=\{p: p$ is an atom of $L(C)$ and $D(p)$ is in $M\}$.

The standard "hereditary set" construction can be modified to give the following result.

LEMMA 2. If $L$ is a lattice with $\# L=n+1$, then $L$ is isomorphic to a join-sublattice of $2^{n}$ containing 0 .

THEOREM. If $L$ is a finite lattice then there is a finite-dimensional convex set $C$ such that $L$ is isomorphic to $D L(C)$.

Since $2^{n}$ is the face lattice of a simplex, the result follows from Lemma 1.

The set $C$ obtained here is of large dimension and highly nonclosed. By imposing additional conditions on $L$, we can reduce the size of $C$. For example if $L$ is coatomistic and has $k$ coatoms, $L$ is isomorphic to $D L(C)$ where $C$ is a subset of the $(k-1)$-dimensional simplex $S$ and in this case the coatoms of $L$ are in $1: 1$ correspondence with the facets of $S$.

\section{BIBLIOGRAPHY}

1. Mary Katherine Bennett, Lattices of convex sets, Trans. Amer. Math. Soc. (to appear).

DEPARTMENT OF MATHEMATICS AND STATISTICS, UNIVERSITY OF MASSACHUSETTS, AMHERST, MASSACHUSETTS 01002

AMS (MOS) subject classifications (1970). Primary 06A20.

Copyright $\odot$ 1977, American Mathematical Society 